\title{
Measurement of the $\boldsymbol{k}$-value in semiconductor structures
}

\author{
P.L. Potapov, H.-J. Engelmann and E. Zschech
}

AMD Saxony LLC \& Co. KG, Wilschdorfer Landstr. 101, D-01109 Dresden, Germany

Continuing down-sizing of $\mathrm{Si}$ integrated circuits requires the application of materials with the precisely controlled dielectric constant ( $\boldsymbol{k}$-value). TEM and STEM combined with Electron EnergyLoss Spectroscopy (EELS) is the promising technique to determine the $\boldsymbol{k}$-value in semiconductor structures on the nanometer scale. Due to the recent development of monochromated TEM instrumentation, it is now possible to collect EELSpectra with high energy resolution and dispersion allowing an accurate analysis of EELSpectra in the range of low energies. Particularly, the band gap can be directly measured from such spectra. The subsequent Kramers-Kronig transformation [1] allows to retrieve the dielectric function $\varepsilon(E)$ and, by interpolating the real part of $\varepsilon$ to zero-energy, the dielectric constant $\boldsymbol{k}$.

$\boldsymbol{k}$-values of advanced materials were extracted from the high energy resolution EELSpectra collected in a monochromated Technai TF20 microscope which provides an energy resolution up to $0.2 \mathrm{eV}$ and an energy dispersion up to $0.01 \mathrm{eV} / \mathrm{chanel}$. The applied procedure for data analysis is based on the Kramers-Kronig transformation and the assumption of the uniform thickness of the investigated area, which is reasonably fulfilled for FIB prepared samples. The Cherenkov radiation might cause dramatic artifacts and must be subtracted from the EELSpectra prior the Kramers-Kronig transformation. The active $\mathrm{Si}(\boldsymbol{k}$ value 11.8) that is present in the majority of semiconductor structures can be taken as a reference to determine the absolute thickness. Examples of the spectra and measured $\boldsymbol{k}$-values for several semiconductor structures are shown in Fig.1-3.

References

[1] R.F. Egerton, Electron Energy-Loss Spectroscopy in the Electron Microscope, Plenum, New York, 1996. 


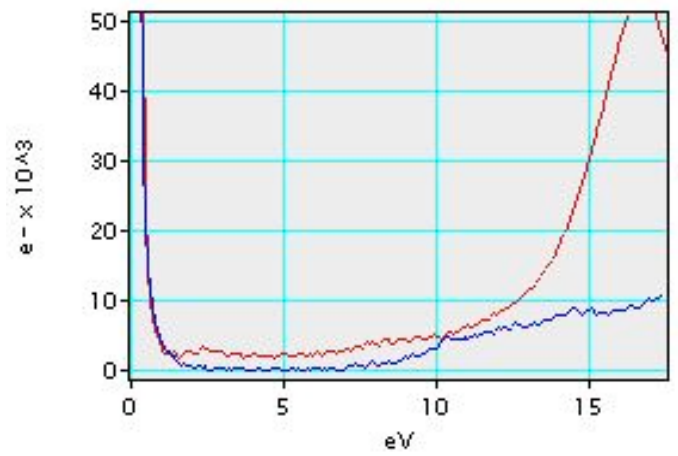

(a)
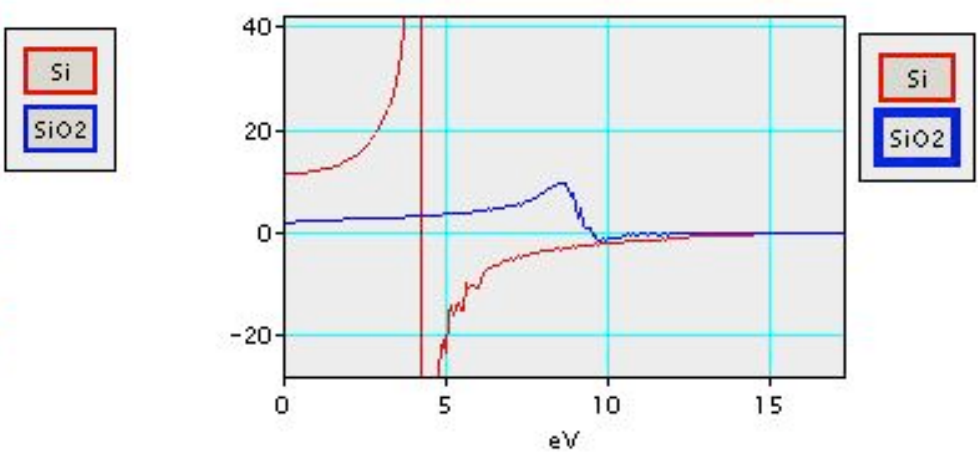

(b)

FIG. 1. (a) Collected EELSpectra and (b) extracted real part $\varepsilon_{1}$ of dielectric function for $\mathrm{Si}$ and $\mathrm{SiO}_{2}$. The band gap can be directly measured from spectra while the $\boldsymbol{k}$-value is found by extrapolating $\boldsymbol{\varepsilon}_{\boldsymbol{l}}$ to zero-energy.

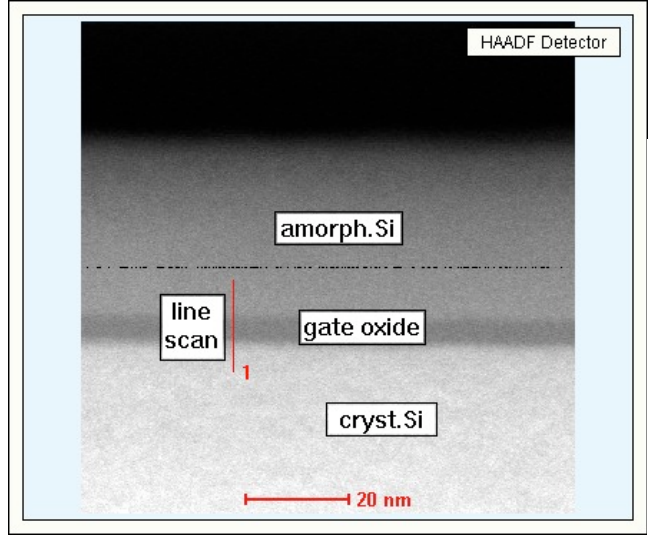

(a)

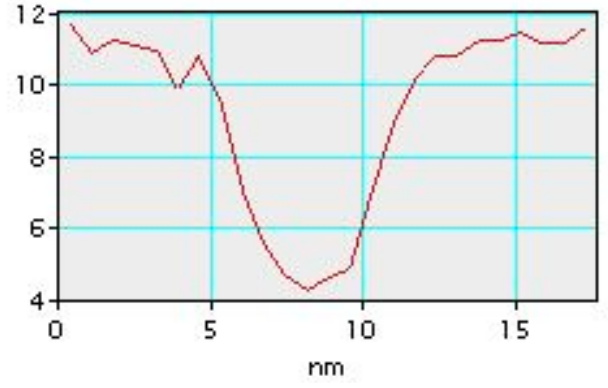

(b)

FIG. 2. (a) STEM image with the indicated line scan through $5 \mathrm{~nm}$ gate oxide layer and (b) $\boldsymbol{k}$-profile along this line scan.

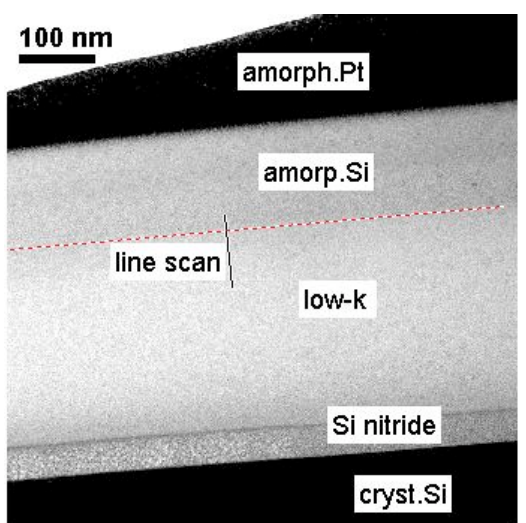

(a)

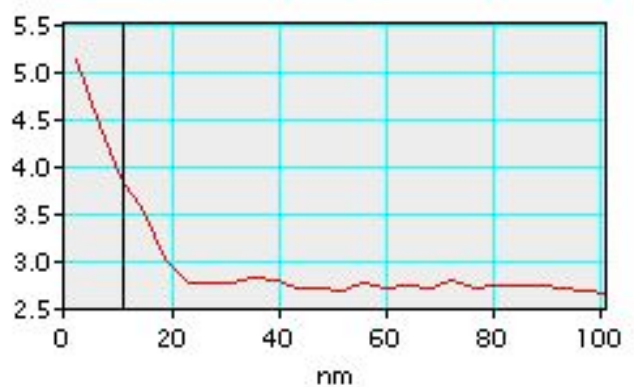

(b)

FIG. 3. (a) TEM image of the low- $k$ layer and (b) $k$-profile along the line scan near the $\mathrm{Si} /$ low- $k$ interface (denoted by the vertical line). The measurements shows clearly the increase of the $\boldsymbol{k}$-value in the outer $\sim 10 \mathrm{~nm}$ of low- $k$ material damaged by plasma treatment. 